\title{
Study and Realization of 3D Visualization System of Power Transmission Grid
}

\author{
Wan Huijiang ${ }^{a}$, Liang Ling ${ }^{b}$, Ma Jianweic, Jiang Zaineng ${ }^{d}$ \\ Guizhou Power Grid Co., Ltd., Power Dispatching Control Center, Guiyang, Guizhou 550002 China \\ a408438504@qq.com, ’361550717@qq.com, c425054771@@qq.com, '991980142@qq.com
}

Keywords: OSG; power transmission grid; 3D visualization; scene roaming; path roaming

\begin{abstract}
With the acceleration of the construction of smart power grid, the power industry has put forward more requirements on the construction, design and operation management of transmission network, and the 3D visualization of transmission grid becomes more and more important. Therefore, in order to show the grid electricity staff such information as the environment and components of transmission grid in a more direct and vivid way, this paper refers to the design of Internet technology and realizes the 3D visualization system of power transmission grid with timeliness and interactivity, which improves the maintenance ability and management of transmission network.
\end{abstract}

\section{Introduction}

In recent years, with the rapid development of power grid enterprise in our country, the accelerating of the construction of the transmission line and the implementation of the aerial photography technology by different transmission owners in the country, have higher requirements on the design, construction, and operation management. The 3D visualization technology of power transmission grid combines the design of transmission line with such information as the landform, landscape, weather conditions and other information and expresses in a vivid and direct way in 3D visualization method, so as to make the observers obtain the needed information in a rapid and accurate way. The design of $3 \mathrm{D}$ visualization system of power transmission grid is one of the challenging and promising directions on the virtual simulation on real-time scenes.

\section{Research on 3D visualization system of power transmission network}

\subsection{Hardware basis of research}

The 3D visualization system of power transmission grid displays technology research and application through the 3D dynamic intelligent technology in real-time and guides the implementation of 3D dynamic technology in grid intelligent display. Through 3D dynamic grid real-time information intelligent displaying technology research and application, the application of 3D display technology, combined with the text, graphics, video, animation, and other forms of power grid dispatching operation data, can conduct the display in a central, dynamic, real-time and themed way.

Through 3D dynamic grid real-time information intelligent display technology research and application, it can resolve such problems as disunity in data, style, dimension, and perspective in different application systems in electric power dispatching control center of Guizhou Power Grid, so as to realize the goal of display integration, panoramic view, and lean management, so as to elevate working efficiency. Table 1. Composition of the graphic processing platform of intelligent display system:

The graphical processing platform of intelligent display system is shown in Table 2:

Hardware equipment working environment conditions: temperature: $-5{ }^{\circ} \mathrm{C} \sim+40{ }^{\circ} \mathrm{C}$; relative humidity: no more than $90 \%$ (there should be no condensation in the device, or freezing); atmospheric pressure: $70 \mathrm{kPa} \sim 106 \mathrm{kPa}$. 
Table 1. Graphic processing platform of intelligent display system

\begin{tabular}{|c|c|}
\hline Items & Index \\
\hline Model & Think Station P910 \\
\hline Operating system & Windows 7 Professional 64 digits \\
\hline CPU & $2 *$ Intel Xeon E5-2699 v4 \\
\hline Memory & 64GB \\
\hline GPU & ATI FirePro ${ }^{\mathrm{TM} W 8100}$ \\
\hline Hard disk system & $1^{*}$ 1TB SATA,10000rpm \\
\hline CD-ROM & 16X DVD-ROM SATA 1st Drive \\
\hline Network card & Integration 10/100/1000M Ethernet \\
\hline
\end{tabular}

Table 2 Database platform of intelligent display system

\begin{tabular}{|c|l|}
\hline Item & \multicolumn{1}{|c|}{ Index } \\
\hline Model & ThinkServer TS250 \\
\hline Operating system & $\begin{array}{l}\text { Windows Server 2008 R2 64 digits (including SQL } \\
\text { SERVER2012 database software) }\end{array}$ \\
\hline CPU & Intel Core 4 E3-1225 v5 3.3Ghz \\
\hline Memory & 32GB \\
\hline Hard disk system & 2*1TB SATA 7200 Raid 1 Disk Array \\
\hline CD-ROM & 16X DVD-ROM \\
\hline Network card & Double 10/100/1000M Ethernet \\
\hline
\end{tabular}

Table 3 Movable touch terminal

\begin{tabular}{|c|l|}
\hline Item & \multicolumn{1}{|c|}{ Index } \\
\hline Model & Apple iPad Air 2 \\
\hline Operating system & ios system \\
\hline CPU & A8X chip \\
\hline DPI & 2048 x 1536 \\
\hline Memory capacity & 64GB \\
\hline Endurance & As long as 10 hours \\
\hline WiFi functions & $\begin{array}{l}802.11 \mathrm{a} / \mathrm{b} / \mathrm{g} / \mathrm{n} \text { WLAN network }(802.11 \mathrm{n} \quad \text { between } 2.4 \mathrm{GHz} \\
\text { and } 5 \mathrm{GHz})\end{array}$ \\
\hline
\end{tabular}

\subsection{System view window design}

In the process of realizing the grid system, it needs to change the position of the camera appropriately to observe the grid better; bind the camera with the user's eyes, and the camera's angle is equivalent to the angle of the eyes. The 3D world and screen pixel changes include multiple types of operations that require the implementation of the $\mathrm{H}$ process transformation.

(1) Viewpoints changes of power transmission grid: the grid model library function called gluLookAt () changes the viewpoint, transforming the scene from the world coordinate system to the camera coordinate system; the function uses $\mathrm{H}$ parameters, viewpoints, reference point locations and the upper location of the point of view to show the camera position. In the world coordinate system, we use 3 score vectors of $n, u$, and $v$ to represent the coordinate basis, and the score vector $\mathrm{W}$ is from the reference point center to the vector of the view eye, which is equivalent to the z-axis of the world coordinate system; $u$ is the cross product of up and $n$ in the direction of the view, equivalent to the $\mathrm{X}$ axis; the score vector $\mathrm{V}$ is the cross product of $\mathrm{n}$ and $\mathrm{u}$, which is equivalent to the $\mathrm{Y}$-axis. The mathematical expressions are: 


$$
n=\frac{\text { eye }- \text { center }}{\mid \text { eye }- \text { center } \mid}, u=\frac{u p \times n}{|u p \times n|}
$$

The basis vectors of the world coordinate system are $X(1,0,0), Y(0,1,0), Z(0,0,1)$, from which we can get an outline excessive matrix A:

$$
A=\left[\begin{array}{cccc}
\mu & v & n & 0 \\
\mu & v & n & 0 \\
\varpi & v & n & 0 \\
-e & -e & -e & 1
\end{array}\right]
$$

(2) Projection: change the scene which has transformed into camera coordinate system to the projected coordinate system again to get the vector usually under the standard coordinates, in which all the coordinates are mapped to - $1-1$, and the value from screen to the far plane $\mathrm{Z}$ is 1 , with the close screen coordinate of 1.

(3) Viewport transformation: this step is to show the projection transformation to the screen, which is similar to printing out the photos. This transformation means that the scene is eventually shifted to the window coordinate system, where the $\mathrm{X} / \mathrm{Y}$ direction of the $\mathrm{X} / \mathrm{Y}$ axis is based on the range of $(0,0)$ - (width, height). The $\mathrm{z}$-axis is going along the screen, and the value scope is $[0,1]$. Realizing these features requires the camera node Camera category, image device object, GraphicsContext class, and the viewfinder osgViewer class provided by OSG. The view finder can organize and manage the nodes in the scene camera, and meanwhile, in combination with the variables and updating of the scene nodes, each frame stored in the camera can be played to complete and show an integrated 3D simulation scenario. The camera node is used to realize the change of view point in the camera, which is mainly through matrix change. The graphic device object defines the device platform in the scene, determines the corresponding relationship between rendering effect and pixel cache, and has many operational functions.

\subsection{The realization of weather effect}

In the 3D visualization system of power transmission grid, the simulation of the weather effects of rain and snow can improve the real sense of the system. In order to realize this function, OSG provides the particle system osgParticl. The effect of particle motion with natural physical characteristics can be simulated effectively. Particle system is a certain number of smile particle collection with simple shape into an irregular fuzzy objects; each particle has size, position, shape, color and other properties, the corresponding methods in the particle system are also provided to achieve it. Through establishing the steps of the particle system, and the basic properties of the self-defined snow particles can be set as shown in table 4 below according to the predefined particle system of OSG:

Table 4 Snow effect data parameter setting

\begin{tabular}{|c|c|l|}
\hline Parameters & Attribute description & Setting values \\
\hline Life Time & Particle life cycle & 3 \\
\hline Size Range & Particle size & $(15000,30000)$ \\
\hline Alpha Range & Particle transparency & $(0.6 \mathrm{f}, \quad 1.0 \mathrm{f})$ \\
\hline Color Range & Particle color & $(1.0 \mathrm{f}, 0.5 \mathrm{f})$ \\
\hline Radius & Particle radius & $0.05 \mathrm{f}$ \\
\hline Mass & Particle quantity & $0.1 \mathrm{f}$ \\
\hline Texture file & Particle material & snow.rgb \\
\hline emissive-particles & Radial pattern & false \\
\hline lighting & Added light & false \\
\hline RateRange & Speed range & Maxvalue 1 \\
\hline ToGravity & Gravitational acceleration & scale=1.0f \\
\hline F1uidToAir & Air attribute & void \\
\hline
\end{tabular}


The process of the weather effect construction in this system is determined according to the steps of the creation of the particle system, it is mainly the simulation operation of the weather snow effect and the rain effect.

\section{Realization of 3D visualization system of power transmission network}

\subsection{Realization of model loading function}

Load the processed model which is constructed and handled by $3 \mathrm{~d}$ Max to the scene, and load the terrain, transmission tower, insulator, and transmission line in accordance with the order into the scene through the VISA Read read Node File () of OSG according to such information as location, size, and direction stored in the database. By default, the right window operation bar control is not selected, and the effect diagram after the successful load is shown in Figure 1, 2.

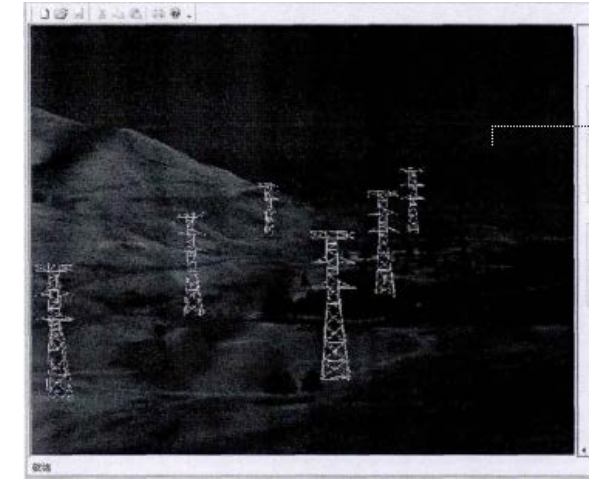

Figure 1 Figure of successful load model

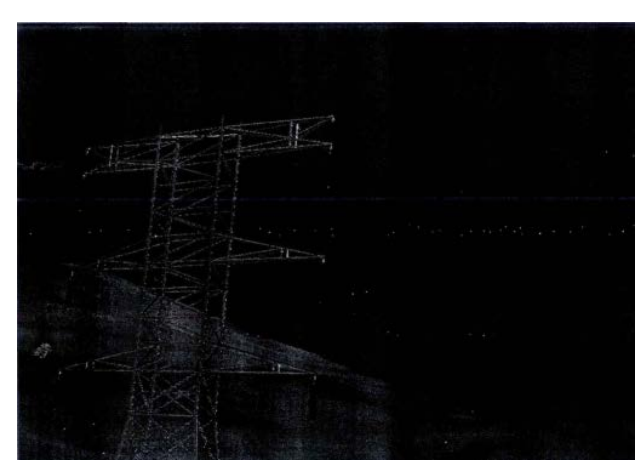

Figure 2 Figure of successful load model

\subsection{The effect of the weather}

(1) The realization of snow effect. In the process of realizing the design of system snow effect, it often combines with fog effect so as to make the scene more realistic; during the operation realization of the snow effect in this system, it is necessary to set the parameters of snow effect in the settings of the menu bar to realize the rendering of snow effect after successful saving. (2) Realization of rain effect. In the implementation process of rain effect, it is similar to the snow effect that particle system is taken to set the corresponding parameters, and the specific operations are the same as the snow effect; the main difference lies in that the material is different. The implementation of rain effect is also accompanied with fog effect. The effect of rain effect is shown in Figure 4 below:

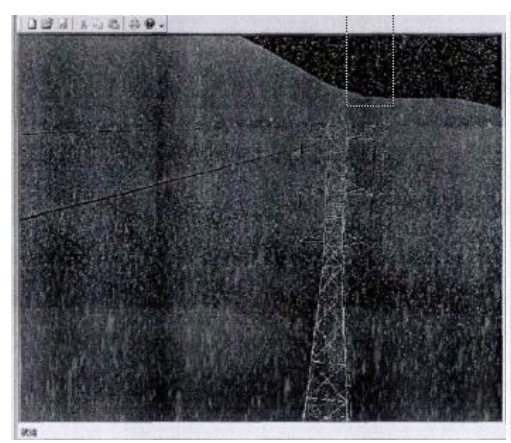

Figure 3 Figure 1 of snow effect realization

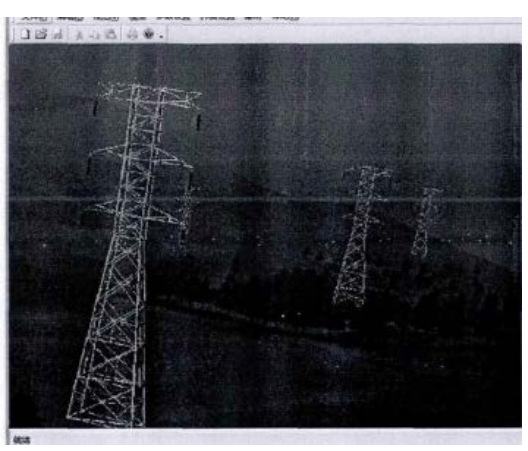

Figure 4 Figure 2 of snow effect realization

\subsection{The realization of roaming}

Roaming has a variety of ways roaming such as scene roaming and path roaming; in the system implementation, the simulation roaming is mainly for roaming roaming and path roaming; the implementation of the bots is realized through smooth and accurate transformation of the observer to the designated position; the view settings of action box of the right child windows of the operating system provides the functions of scene roaming; the observer can realize the results as is 
shown in Figure 5 through moving the button left to right, up and down, front to back to adjust the position:

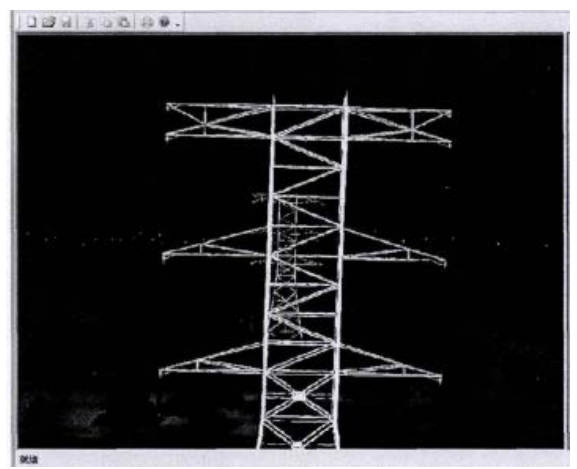

Figure 5 Scene roaming roaming

\section{Conclusions}

With the acceleration of the construction of smart power grid, the research of transmission grid has become more and more, and the 3D visualization of transmission network has become an important subject for the research of power personnel. Therefore, in accordance with the current research trend and in order to display such information as the environment and components of power transmission grid to the electricity staff, this paper designs and realizes the 3D visualization system of power transmission grid; the system has timeliness and interactivity, which can improve the ability of the maintenance of the grid and the management level. The implementation of OSG $3 \mathrm{D}$ image rendering engine and MFC interface technology, can realize the 3D visualization system of transmission network.

\section{References}

[1] Deng Yimin, Tang Zhiqian, Li Hongbing, Yang Zhongya. Real-time Rendering Algorithm Optimization of Large-scale Power Transmission Line Scene Based on LOD [J]. Computer and Modernization, 2017,01:115-118.

[2] Zheng Haoyong, Gaolei. Study on the Construction and Technology Realization of 3D Power Transmission Grid GIS [J]. Science and Technology Information, 2016,36:1-3.

[3] Yu Rongjiang, Zhou Di, Wang Guoliang, Ruan Jin. Application of 3D Panoramic Technology in Power Transmission Grid Planning and Design [J]. Science and Technology Innovation Herald, 2016,35:19-20.

[4] Wang Tingsong, Miao Shuaingxi. Application Study of Power Line Inspection Based on 3D Geoinformation Technology [J]. Geomatics \& Spatial Information Technology, 2016, 09: 137-139 +142 .

[5] Jia Leiliang, Shen Weihua, Ye Kuan, Zhao Xueqian. Grid Simulation Technique Study Based on 3D GIS [J]. Science and Technology Information, 2015,18:51-52. 